\title{
STABILITY PROPERTIES OF A CLASS OF ATTRACTORS
}

BY

\author{
JORGE LEWOWICZ
}

\begin{abstract}
Let $A$ be an attractor of an analytical dynamical system defined in $R^{n} \times R$. The class of attractors considered in this paper consists of those $A$ which remain stable as invariant subsets of the complex extension of the flow to $C^{n} \times R$. If $A$ is a critical point or a closed orbit, these are the elementary or generic attractors. It is shown that such an $A$ is always a submanifold of $R^{n}$ and that there exists a Lie group acting on $A$ and containing the given flow as a one parameter dense subgroup; as a consequence, some necessary and sufficient conditions for an analytical dynamical system to have an attracting generic periodic motion are given.

It is also shown that for any flow $C^{\prime}$-close to the given one, there is a unique retraction of a neighbourhood of $A$ onto a submanifold of $R^{n}$ homeomorphic to $A$ that commutes with the flow.
\end{abstract}

1. In this paper we study a family of attractors of an analytic flow taking place in $R^{n}$ whose properties are, in a certain sense, similar to those of the simplest attractors.

Let $\Omega$ be an open connected subset of $R^{n}$ and $X: \Omega \rightarrow R^{n}$ an analytic vector field defining the analytic flow $\phi$ on an open subset of $\Omega \times R$. A compact $\phi$ invariant set $A \subset \Omega$ is an attractor if:

(1) for any neighbourhood $U \subset \Omega$ of $A$, there is another neighbourhood $V$, such that $x \in V$ implies $\phi(x, t) \in U$ for $t \geq 0$, and

(2) there is a neighbourhood of $A$ with the property that if $x$ belongs to it, $\lim _{t \rightarrow \infty} \operatorname{dist}(\phi(x, t), A)=0$.

Since $X$ is analytic there is a complex extension of $X$ to a $C^{n}$-neighbourhood $W$ of $A$. We consider here the attractors $A$ which are stable as invariant subsets of the flow generated be this extension of $X$, or, in other words, we consider those $A$ that satisfy property (1) with respect to the analytic extension of $\phi$ to an open subset of $W \times R$. (1) For brevity, let us denote also by $\phi$ this extension and call $C$-generic these attractors. It is not reasonable to expect an attractor $A$ to be also an attractor of the extended flow: if $A$ contains a periodic motion $x$, say of minimum period $\tau>0$, then for small real $s, \phi(x, s i)$ lies close to $A$ but not on it and $\phi(\phi(x, s i), \tau)=\phi(x, \tau+s i)=\phi(x, s i)$ since the equality $\phi(x, t+\tau)=\phi(x, t)$ holds for real $t$; hence, condition (2) is not satisfied. One of the motivations for studying this class of attractors comes from the fact that in the simplest cases the

Received by the editors January 30, 1970 and, in revised form, April 20, 1972.

AMS (MOS) subject classifications (1970). Primary 34C25, 34C30, 34D35; Secondary 34C35, 58F15, $58 \mathrm{Gl} 10$.

Key words and phrases. Analytical dynamical system, stable invariant set, elementary or generic attractors, complex Poincaré diffeomorphism, complex extension of the flow, retraction commuting with the flow, minimal set, one parameter subgroup, Banach manifold, contraction.

(1) The parameter $t$ of the flow $\phi$ will always be assumed to be real unless otherwise stated. 
$C$-generic attractors are exactly the generic attractors defined in $[5, \S 2$, Theorem 1].

We prove, among other things, that a $C$-generic attractor is always an analytical manifold, and construct a compact Lie group acting on it and containing the given flow as a one parameter dense subgroup. As a corollary we get some characterizations of analytic dynamical systems possessing a generic attracting periodic motion. Also, we get several results on the perturbation theory of $C$-generic attractors by studying the action of the flow on a Banach manifold of retractions of $\Omega$.

Finally, perhaps it is convenient to say that most of the results can be extended in several ways, for instance replacing $R^{n}$ by a differentiable manifold and the analytic flow by a $C^{1}$-flow, but since we believe that these generalizations will not add anything of a conceptual character, we prefer to keep in the abovementioned context, where some of the results are richer and the techniques simpler.

2. We begin by studying the simplest attractors, i.e., equilibrium points and closed orbits.

Theorem 1. Let $A$ be an attractor for an analytic flow in $R^{n}$ and assume that $A$ is a periodic motion or a critical point. Then, $A$ is generic [5] if and only if it is $C$ generic.

Proof. We give the proof for $A$ a periodic motion; the case of a critical point can be handled with similar arguments.

We assume, as we may, that $A$ goes through the origin 0 in $R^{n}$ and that the vector field $X$ takes at this point the value $X(0)=(0, \ldots, a)$ where $a \neq 0$. Let $\tau>0$ be the fundamental period of $A$ and $\phi=\left(\phi_{1} \ldots \phi_{n}\right)$ the flow defined by $X$. Consider the equation

$$
\phi_{n}(x, t)=0
$$

for $x$ in a complex neighbourhood of 0 and $t$ in a complex neighbourhood of $\tau$. Applying the implicit function theorem we get another complex neighbourhood of 0 and a positive number $\varepsilon$ such that, for $x$ in that neighbourhood, equation (1) has exactly one complex root $t(x)$ satisfying $|t(x)-\tau|<\varepsilon$. For points $x$ $=\left(x_{1}, \ldots, x_{n}\right)$ close to 0 and lying on the hyperplane $H=\left\{x \in C^{n}: x_{n}=0\right\}$, we consider the complex analytic Poincaré mapping $\psi: H \rightarrow H$ defined by $\psi(x)=\phi(x, t(x))$.

To prove the necessity, call $T: H \rightarrow H$ the linear part of $\psi$ at the origin which, by assumption, has proper values $\lambda_{i}: i=1,2, \ldots, n-1$, satisfying the inequalities $0<\left|\lambda_{i}\right|<1$, and take a norm \|\| for $H$, such that the corresponding $\|T\|<1$ (see [5, p. 102]). 
If we write $\psi(x)=T x+\psi_{0}(x)$, then

$$
\lim _{\|x\| \rightarrow 0} \frac{\psi_{0}(x)}{\|x\|}=0
$$

and we may choose a complex neighbourhood of 0 in $H$ such that if $x$ belongs to it, $\left\|\psi_{0}(x)\right\|<\frac{1}{2}(1-\|T\|)\|x\|$. Thus for these $x,\|\psi(x)\| \leq\left(\|T\|+\frac{1}{2}(1-\|T\|)\right)$ - $\|x\|=\mu\|x\|$, where $0<\mu<1$. Therefore if $x$ is small enough, the $n$-iterate of $\psi, \psi^{n}$, is defined at $x$ for $n=1,2, \ldots$, and $\left\|\psi^{n}(x)\right\| \leq \mu^{n}\|x\|$.

To prove that for real $t \geq 0, \phi(x, t)$ is close to $\phi(0, t)$, let us write

$$
\begin{aligned}
\phi(x, n \tau) & =\phi\left(\phi\left(x, \sum_{1}^{n} t\left(\psi^{i-1}(x)\right)\right), \sum_{1}^{n}\left(\tau-t\left(\psi^{i-1}(x)\right)\right)\right) \\
& =\phi\left(\psi^{n}(x), \sum_{1}^{n}\left(\tau-t\left(\psi^{i-1}(x)\right)\right)\right),
\end{aligned}
$$

and notice that, since $t(x)$ depends analytically on $x$,

$$
\left|\sum_{1}^{n}\left(\tau-t\left(\psi^{i-1}(x)\right)\right)\right| \leq \sum_{1}^{n} M\left\|\psi^{i-1}(x)\right\| \leq M\|x\| \sum_{1}^{n} \mu^{i-1} .
$$

From these remarks follows easily the asserted stability of the periodic motion as an invariant subset of the extended flow.

Now we prove the sufficiency. The functions $\phi_{l}, \phi_{t}(x)=\phi(x, t)$, are defined, analytic and uniformly bounded for $t \geq 0$ on a suitable $C^{n}$-neighbourhood of 0 ; using Cauchy's formula we may show that the derivatives $\left(\partial \phi_{i} / \partial x_{j}\right)(x, t)$, $i=1, \ldots, n, j=1, \ldots, n$, are also uniformly bounded for $t \geq 0$ in a smaller $C^{n}$-neighbourhood of 0 . This implies, in particular, the positive Lyapunov stability of the periodic motion. Let $U$ be a $C^{n}$-neighbourhood of 0 and $\varepsilon$ a positive real number so that for $x \in U, \phi_{n}(x, t)=0$ has only one root $t(x)$ with $|t(x)-\tau|<\varepsilon$. Because of the Lyapunov stability, and since $\phi(0, n \tau)=0, n \geq 0$, we may choose a neighbourhood of 0 in $H$, say $V$, such that $\phi(x, n \tau) \in U$ for $x \in V, n=0,1,2, \ldots$ Consequently, there are complex numbers $z_{n}, n=1$, $2, \ldots$, such that for these $n, \phi\left(x, n \tau+z_{n}\right) \in H$, and $\left|z_{n}\right|<\varepsilon$. It is clear then that if $\varepsilon$ is small enough, $\psi^{n}(x)=\phi\left(x, n \tau+z_{n}\right)$, and therefore 0 is a positively stable fixed point of the complex Poincare diffeomorphism $\psi$.

Let $V$ be a neighbourhood of 0 in the complex subspace $H$ such that the $\psi^{n}$, $n=1,2, \ldots$, are defined and equiuniformly bounded on $V$; since the $\psi^{n}$ constitute there a normal family, there is a subsequence $\psi^{n_{k}}$ that converges uniformly on $V$ to an analytic funcion. It follows easily from the assumptions that if $x$ is real and sufficiently small, $\lim _{n \rightarrow \infty} \psi^{n}(x)=0$, which implies readily that $\psi^{n_{k}} \rightarrow 0$ uniformly on $V$. Consequently, there is a $\delta>0$ and a positive integer $N$ so that if $x \in H,\|x\| \leq \delta$, then $\left\|\psi^{N}(x)\right\|<\delta$, and $\psi^{n}(x) \in V$, $n=1,2, \ldots, N$. This situation cannot be changed by small analytic perturbations of $\psi$, i.e. if $\psi^{\prime}$ is close enough to $\psi$ on $V$ and $\psi^{\prime}(0)=0, \psi^{\prime N}$ will have the 
same property as $\psi$ with respect to the complex ball $\{x \in H:\|x\| \leq \delta\}$.

On the other hand, this property implies that all the iterates $\psi^{\prime n}, n=1,2, \ldots$, are equiuniformly bounded on that ball, and as we remarked before, this in turn implies the positive stability of 0 under the discrete flow defined by $\psi^{\prime}$. Then if the linear part of $\psi$ had some proper value of absolute value 1, we could add to $\psi$ a small linear transformation of $H$ to get an analytic diffeomorphism $\psi^{\prime}$ close to $\psi$ and having 0 as an unstable fixed point [5]. This completes the proof.

3. In this section we prove several results of a topological character concerning general $C$-generic attractors.

Let $U \subset R^{n}$ be a compact neighbourhood of the $C$-generic attractor $A$ with the property that for $x \in U, \lim _{t \rightarrow \infty} \operatorname{dist}(\phi(x, t), A)=0$, and such that there exists $W$, a complex $C^{n}$-neighbourhood of $U$, having all its iterates $\phi(W, t), t \geq 0$, uniformly bounded. The analytic functions $\phi_{t}, t \geq 0$, constitute a normal family on $W$.

Lemma 1. For every $\varepsilon>0$ there exists $\delta>0$ such that, for $x, y \in A,\|x-y\|$ $\leq \delta$ implies $\|\phi(x, t), \phi(y, t)\| \leq \varepsilon$ for $|t|<\infty$.

Proof. As a consequence of Cauchy's formula and the compactness of $U$ we have that for every $\varepsilon>0$ there exists $\delta>0$ such that $x, y \in U$ and $\|x-y\| \leq \delta$ imply $\|\phi(x, t)-\phi(y, t)\| \leq \varepsilon$ for $t \geq 0$. On the other hand, it is clear that if $x=\lim \left(\phi\left(x_{0}, t_{n}\right)\right), y=\lim \left(\phi\left(y_{0}, t_{n}\right)\right), \lim t_{n}=+\infty$, and $\left\|\phi\left(x_{0}, t\right)-\phi\left(y_{0}, t\right)\right\|$ $\leq \varepsilon$ for $t \geq 0$, then, for each $t \in R,\|\phi(x, t)-\phi(y, t)\| \leq \varepsilon$.

Now choose a sequence $\phi_{t_{n}}, t_{n} \rightarrow \infty$, such that $\phi_{t_{n}}$ converges uniformly to a continuous mapping $\theta: U \rightarrow U$. Because of the way $U$ was chosen, $\theta(U) \subset A$; on the other hand it follows from the compactness of $U$, the fact that, for each $n, \phi_{t_{n}}(U) \supset A$ and the uniform convergence of the $\phi_{t_{n}}$, that $\theta(U) \supset A$.

Thus $\theta(U)=A$. Then for each $x \in A, \theta^{-1}(x)$ is a compact nonvoid subset of $U$, and it is easy to show that if $V$ is any neighbourhood of $\theta^{-1}(x), \theta(V)$ is an $A$ neighbourhood of $x$. It follows from this and the previous remarks that given $\varepsilon>0$ and $x \in A$ there exists an $A$-neighbourhood of $x$ such that if $y$ and $z$ belong to it, $\|\phi(y, t)-\phi(z, t)\| \leq \varepsilon, t \in R$, and the thesis of the lemma is now an immediate consequence of the compactness of $A$.

Let $V$ be an open positively invariant neighbourhood of $A, V \subset U$.

Lemma 2. There exists one and only one analytic retraction $\alpha$ of $V$ onto $A$ that commutes with the flow, i.e. such that $\alpha_{x}^{\prime} X_{x}=X_{\alpha(x)}$.

Proof. Let $t_{n}$ be a sequence such that $t_{n} \rightarrow \infty, t_{n}-t_{n-1} \rightarrow \infty$ and $\phi_{t_{n}}$ converges uniformly on $U$. Then, if $s_{k}$ is a subsequence of $t_{n}-t_{n-1}$ such that $\phi_{s_{k}}$ converges uniformly on $V$ to an analytic function $\alpha$, we have clearly that $\alpha(V)=A$, and since for $n$ large enough $\left\|\phi_{t_{n+1}}(x)-\phi_{t_{n}}(x)\right\|$ is arbitrarily small, uniformly for all $x \in A$, we get from Lemma 1 that $\left\|\phi_{s_{k}}(x)-x\right\|$ is also uniformly small for $k$ large. 
Therefore $\alpha(x)=x$ for $x \in A$ and $\alpha$ is an analytic retraction of $U$ onto $A$. Obviously, $\alpha$ commutes with each $\phi_{t}$.

Let $x \in V$, then $\|\phi(x, t)-\phi(\alpha(x), t)\|=\|\phi(x, t)-\alpha(\phi(x, t))\|$ and as $\lim _{t \rightarrow \infty} \operatorname{dist}(\phi(x, t), A)=0$ and $\alpha$ is a retraction onto $A$, it follows that $\lim _{t \rightarrow \infty}\|\phi(x, t)-\phi(\alpha(x), t)\|=0$.

Now we prove the uniqueness of $\alpha$ by showing that $\alpha(x)$ is the only point in $A$ with that property. In fact, the existence of another point in $A$ with the same property would contradict Lemma 1.

Lemma 3. Let $V \subset R^{n}$ be an open set, $A$ a compact subset of $V$ and $\alpha$ an analytic retraction of $V$ onto $A$. Then $A$ is an analytic submanifold of $R^{n}$.

Proof. Let $k=\max _{x \in V}\left\{\right.$ rank $\left.\alpha^{\prime}\right\}$; as the case $k=0$ is trivial, we may assume $k>0$. Take $x \in V$ such that rank $\alpha_{x}^{\prime}=k$; since $\alpha \circ \alpha=\alpha, \alpha_{\alpha(x)}^{\prime} \circ \alpha_{x}^{\prime}=\alpha_{x}^{\prime}$, and it follows that $\alpha^{\prime}$ has maximum rank at $\alpha(x)$ and hence in a neighbourhood of it. From well-known results in calculus we get that there exists an $R^{n}$. neighbourhood $V$ of $\alpha(x)$ such that $\alpha(V)$, as a subspace of $R^{n}$, is analytically homeomorphic to an open subset of $R^{k}$. On the other hand $V \cap A=\alpha(V \cap A)$ $\subset \alpha(V)$, and since $\alpha(V) \subset A, V \cap A=V \cap A \cap \alpha(V)=V \cap \alpha(V)$. Consequently, $\alpha(x)$ has an $A$-neighbourhood analytically homeomorphic to an open subset of $R^{k}$.

The set $B=\left\{y \in A\right.$ : $\left.\operatorname{rank} \alpha_{y}^{\prime}=k\right\}$ is open in $A$; let us show that $B$ is also closed. If $x_{n}$ is a sequence in $B$ converging to $x \in A$, and $\left\{u_{n}^{1}, \ldots, u_{n}^{k}\right\}$ an orthonormal system in the range of $\alpha_{x_{n}}^{\prime}, n=1,2, \ldots$, such that $u_{n}^{i} \rightarrow u^{i}$, $i=1, \ldots, k$, we have, since for all $n, \alpha_{x_{n}}^{\prime} u_{n}^{i}=u_{n}^{i}$, that $\alpha^{\prime}$ has maximum rank at $x$ as we wanted to show. Since $A$ is connected, $B=A$, and on account of the previous remark, this completes the proof.

Theorem 2. A compact connected subset of $R^{n}$ is a $C$-generic attractor of some analytic flow in $R^{n}$ if and only if it is an analytic submanifold of $R^{n}$.

Proof. The necessity follows from the lemmas. For the sufficiency, consider an analytic submanifold $A \subset R^{n}$ and the function $r: R^{n} \rightarrow A$ defined by $r(x)$, where

$$
\|x-r(x)\|=\min _{y \in A}\|x-y\| .
$$

If $x$ is close enough to $A$, it is known (see [2, p. 38]) that $r(x)$ is well defined and the function $r$ is analytic in a suitable $R^{n}$-neighbourhood of $A$. Moreover, if $x \in A$ and if $u$ is small and normal to $A$ at $x, r(x+u)=x$. Consequently the analytic vector field $x \rightarrow r(x)-x$ defined on that neighbourhood determines an analytic flow $\phi$, such that $\phi(x, t)=r(x)+(x-r(x)) e^{-t}$. From this formula it is easy to obtain that $A$ is stable as an invariant subset of the $C^{n}$-extension of $\phi$.

4. Now we are going to study the flow on $C$-generic attractors and to associate to each a Lie group acting on it.

Let $A$ be a $C$-generic attractor; it follows easily from Lemma 1 that if $x \in A$, the closure of the trajectory through $x$ is a minimal set $M_{x}$. So $A$ is a union of 
disjoint minimal sets. Let $G$ be the closure of the set of analytic homeomorphisms $\left\{\phi_{t} / A: t \in R\right\}$ considered as a subspace of the space of mappings from $A$ into $A$ with the topology of uniform convergence on $A$. Since there exists a sequence $t_{n} \rightarrow \infty$ such that $\phi_{t_{n}} / A$ converges uniformly to the identity (Lemma 2 ), $G$ is also the closure of the set $\left\{\phi_{t} / A: t \geq 0\right\}$ and inasmuch as the $\phi_{t}, t \geq 0$, constitute a normal family when restricted to a suitable $C^{n}$-neighbourhood of $A$, we may deduce easily that each element of $G$ is analytic and that $(G, \circ)$ with the relative topology is a compact connected abelian group such that each $M_{x}$ is homeomorphic to a factor group of $G$.

Theorem 3. Let $k$ be the highest of the dimensions of the minimal sets contained in $A$. Then $G$ is a k-dimensional torus.

Proof. Since $G$ is a group acting effectively by analytic mappings on the manifold $A$, it is a Lie group (see [3, p. 208]) and, being abelian compact and connected, it is a torus. To prove that $G$ is $k$-dimensional, choose $x_{0}$ such that $\operatorname{dim} M_{x_{0}}=k$ and call $G_{x_{0}}$ the subgroup of $G, G_{x_{0}}=\left\{\alpha \in G: \alpha\left(x_{0}\right)=x_{0}\right\}$. Clearly, $G_{x_{0}}=G_{x}$ for each $x \in M_{x_{0}}$. The mapping $[\gamma] \rightarrow \gamma\left(x_{0}\right)$ from $G / G_{x_{0}}$ to $M_{x_{0}}$ is a homeomorphism and $M_{x_{0}}$ gets in this way a Lie group structure. Let $U$ be a neighbourhood of $x_{0}$ in $M_{x_{0}}$ such that it contains no nontrivial subgroups. We may choose $\delta>0$ so small that if $x \in A, \gamma \in G,\left\|x-x_{0}\right\| \leq \delta$ and $\gamma(x)=x$, then $\gamma^{n}\left(x_{0}\right) \in U$ for every integer $n$, and therefore $\gamma\left(x_{0}\right)=x_{0}$. Consequently if $\left\|x-x_{0}\right\| \leq \delta, G_{x} \subset G_{x_{0}}$; hence $\operatorname{dim} M_{x}=k$, and it follows that $G_{x_{0}} / G_{x}$ is finite. Thus for each $x \in A$ with $\left\|x-x_{0}\right\| \leq \delta$ there is a positive integer $n(x)$ such that $\gamma \in G$ and $\gamma\left(x_{0}\right)=x_{0}$ imply $\gamma^{n(x)}(x)=x$. By means of a simple category argument we may now show that there is an open subset of $A$, say $D$, and a positive integer $N$ such that $\gamma^{N}(x)=x$ for every $x \in D$, provided that $\gamma\left(x_{0}\right)=x_{0}$. Since each $\gamma \in G$ is analytic, it follows that if for some $\gamma \in G$, $\gamma\left(x_{0}\right)=x_{0}$, then $\gamma^{N}$ is the identity mapping of $A$. Thus, being a Lie group, $G_{x_{0}}$ has to be finite, which implies clearly that $\operatorname{dim} G=k$.

Let $a, b$ be real numbers and let $r$ and $w$ stand respectively. for $x_{1}^{2}+x_{2}^{2}$ and $(r-1)^{2}+x_{3}^{2} ;$ the vector field

$$
\begin{aligned}
& \frac{1}{2}\left(-2 b x_{2}-a x_{1} x_{3} r^{-1}-x_{1} r^{-1}(r-1)\left(w-\frac{1}{4}\right)\right) \partial / \partial x_{1} \\
& \quad+\frac{1}{2}\left(2 b x_{1}-a x_{3} x_{2} r^{-1}-(r-1) r^{-1} x_{2}\left(w-\frac{1}{4}\right)\right) \partial / \partial x_{2} \\
& \quad+\left(a(r-1)-x_{3}\left(w-\frac{1}{4}\right)\right) \partial / \partial x_{3}
\end{aligned}
$$

is defined in some open subset of $R^{3}$ and admits the torus $w=\frac{1}{4}$ as a $C$-generic attractor. The flow $\phi$ can be given explicitly:

$$
\begin{aligned}
\phi(x, t)=\left((r(t))^{1 / 2} \cos \left(b t+\psi_{0}\right),(r(t))^{1 / 2} \sin \left(b t+\psi_{0}\right),\right. \\
\left.w^{1 / 2} \sin \left(a t+\Phi_{0}\right)\left(4 w+(1-4 w) \exp -\frac{1}{2} t\right)^{-1 / 2}\right) ;
\end{aligned}
$$


here $r(t)=1+\left(w^{1 / 2} \cos \left(a t+\Phi_{0}\right)\left(4 w+(1-4 w) \exp -\frac{1}{2} t\right)^{-1 / 2}\right.$ and $\cos \Phi_{0}=$ $(r-1) / w^{1 / 2}, \sin \Phi_{0}=x_{3} / w^{1 / 2}, \cos \psi_{0}=x_{1} / r^{1 / 2}$ and $\sin \psi_{0}=x_{2} / r^{1 / 2}$. If $a / b$ is irrational the group $G$ associated to the attractor is of dimension 2 .

The vector field $\left(-x_{2}-x_{1} \rho\right) \partial / \partial x_{1}+\left(x_{1}-x_{2} \rho\right) \partial / \partial x_{2}-x_{3} \rho \partial / \partial x_{3}$, where $\rho$ $=x_{1}^{2}+x_{2}^{2}+x_{3}^{2}-1$, has the unit sphere of $R^{3}$ as a $C$-generic attractor, and the group $G$ associated to it is isomorphic to $R / Z$ ( $Z$ denotes the integers). All the motions on $\rho=0$ are periodic and except for the poles all have fundamental period 1 . If we consider the product of the flow defined by this vector field and a flow in $R^{2}$ having a periodic motion of fundamental period $\frac{3}{4}$ as a $C$-generic attractor, we get a product flow that has a $C$-generic attractor with a onedimensional group $G$. All the minimal sets on the attractor are one-dimensional and in some of them $G$ does not act effectively.

Theorem 4. Let $A$ be a $C$-generic attractor of the flow $\phi$ defined by the analytic vector field $X$ such that the group $G$ associated to $A$ and $X$ has dimension greater than one. Then there is a $C^{n}$-neighbourhood $U$ of $A$ such that for every $\varepsilon>0$ there exists another analytic vector field $X^{\prime}$ defined in $U$ with the following properties:

(1) $\left\|X_{x}-X_{x}^{\prime}\right\| \leq \varepsilon$ for every $x \in U$.

(2) $A$ is a $C$-generic attractor for $X^{\prime}$ and the retractions for $X$ and $X^{\prime}$ defined by Lemma 2 are the same when restricted to $U$.

(3) The group $G^{\prime}$ associated to $A$ and $X^{\prime}$ is one dimensional.

Proof. The retraction $\alpha$ defined for $X$ by Lemma 2 may be extended to a small $C^{n}$-neighbourhood $W$ of $A$; let us call $\beta$ this extension. The set $B=\{x$ $\in W ; \beta(x)=x\}$ satisfies $B \cap R^{n}=A$ since $\beta / R^{n}=\alpha$.

As for $x \in A, R\left(\alpha_{x}^{\prime}\right) \cap N\left(\alpha_{x}^{\prime}\right)=\{0\}\left(R\left(\alpha_{x}^{\prime}\right)\right.$ and $N\left(\alpha_{x}^{\prime}\right)$ denote respectively the range and null-space of $\left.\alpha_{x}^{\prime}\right)$, we have that for $x$ on a certain $C^{n}$-neighbourhood $V \subset W$ of $A$, the linear subspaces of $C^{n}, R\left(\beta_{x}^{\prime}\right)$ and $N\left(\beta_{x}^{\prime}\right)$, have also a trivial intersection and therefore, for each $u$ tangent to $B$ at $\beta(x)$, the intersection

$$
\left\{\beta_{x}^{\prime-1}(u)\right\} \cap\left\{v \in C^{n}: v=X_{x}+r, r \in R\left(\beta_{x}^{\prime}\right)\right\}
$$

consists of exactly one point. It is easy to show that if $\bar{X}$ is an analytic vector field on $B \cap V$, the vector field $X^{\prime}$ defined on $\left\{\beta^{-1}(B \cap V)\right\} \cap V$ by $X_{x}^{\prime}$ $\in\left\{\beta_{x}^{\prime-1}\left(\bar{X}_{\beta(x)}\right)\right\} \cap\left\{v \in C^{n}: v=X_{x}+r, r \in R\left(\beta_{x}^{\prime}\right)\right\}$ is also analytic and $X^{\prime} / A$ $=\bar{X} / A$.

Take $U \subset V$ to be a compact $C^{n}$-neighbourhood of $A$ so that $\beta(U)$ is invariant under the complex extension of $\phi,\left\|\phi_{t}(x)-\beta \circ \phi_{t}(x)\right\|$ converges uniformly to 0 on $U$, and that, for some $\delta>0$,

$$
U=\left\{\beta^{-1}(\beta(U))\right\} \cap\left\{x \in C^{n}:\|\beta(x)-x\| \leq \delta\right\} .
$$

Let $T>0$ be such that for $x \in U,\left\|\phi_{T}(x)-\beta \circ \phi_{T}(x)\right\| \leq \delta / 2$. If $X^{\prime}$ is another analytic vector field on $U$ that commutes with $\beta$ and such that $\beta(U)$ is also an invariant subset of the flow $\phi^{\prime}$ defined by $X^{\prime}$, then if $X^{\prime}$ is chosen so close 
to $X$ that $\left\|\phi_{T}^{\prime}(x)-\beta \circ \phi_{T}^{\prime}(x)\right\| \leq \delta$, we get that $\phi_{T}^{\prime}(U) \subset U$ for, on the other hand, $\beta \circ \phi_{T}^{\prime}(U)=\phi_{T}^{\prime}(\beta\{U\})=\beta(U)$. Hence, $X^{\prime}$ has a $C$-generic attractor contained in $U$ and containing $A$.

Choose in $G$ a one parameter subgroup $G^{\prime}$ isomorphic to $R / Z$ and close enough to $\left\{\phi_{t}:|t|<\infty\right\}$. $G^{\prime}$ defines a flow and an analytic vector field $\bar{X}$ on $A$. If we define $X^{\prime}$ on $U$ as above we have that $X^{\prime}$ is close to $X$ on $U$ and that every compact $X$ invariant subset of $U$ is also $X^{\prime}$ invariant. Therefore the previous remarks apply to $X^{\prime}$ and we get that $X^{\prime}$ has a $C$-generic attractor $A^{\prime}, A \subset A^{\prime}$ $\subset U$. Since $A \neq A^{\prime}$ implies $\operatorname{dim} A^{\prime}>\operatorname{dim} A$, our result follows easily from the next lemma and the fact that if $X^{\prime}$ is close enough to $X$ there exists an $X$ and $X^{\prime}$ positively invariant neighbourhood of $A$ and $A^{\prime}$.

Lemma 4. Let $A$ be a $C$-generic attractor of the analytic flow $\phi$. Then $A$ is a deformation retract of each sufficiently small positively invariant $R^{n}$-neighbourhood of it.

Proof. Let $U$ be as above and $\alpha$ the retraction of $U \cap R^{n}$ onto $A$. Take $V \subset U \cap R^{n}$ to be a positively invariant neighbourhood of $A$ and assume that the sequence $\left\{\phi_{l_{n}}, n=1,2, \ldots\right\}$ converges uniformly to $\alpha$ on $U$.

Let $\varepsilon>0$ be small enough that for each $x \in A$, the $\varepsilon$-ball centered at $x$ lies in $V$, and choose a positive integer $N$ with the property that $\left\|\phi_{t_{k}}(x)-\alpha(x)\right\| \leq \varepsilon$ for each $x \in V$ and each $k \geq N$. Now we define the retraction $H: V \times[0,1] \rightarrow V$ by

(i) $H(x, u)=\phi(x, u /(1-u))$ for $x \in V, 0 \leq u \leq t_{N} /\left(1+t_{N}\right)$.

(ii) $H(x, u)=\left(1-s_{k}(u)\right) \phi\left(x, t_{k}\right)+s_{k}(u) \phi\left(x, t_{k+1}\right)$ for $x \in V, t_{k} /\left(1+t_{k}\right) \leq u$ $\leq t_{k+1} /\left(1+t_{k+1}\right), k \geq N$. Here

$$
s_{k}(u)=\left(u-\frac{t_{k}}{1+t_{k}}\right)\left(\frac{t_{k+1}}{1+t_{k+1}}-\frac{t_{k}}{1+t_{k}}\right)^{-1} .
$$

(iii) $H(x, 1)=\alpha(x), x \in V$.

5. Let $X$ be an analytic vector field defined on an open subset $\Omega \subset R^{n}$. To state shortly some corollaries of the previous results, let us call an open subset $D \subset \Omega$ admissible if

(i) $D$ is positively invariant under the flow of $\phi$ defined by $X$;

(ii) $X_{x} \neq 0$ for every $x \in D$;

(iii) the integral homology groups of $D$ are those of $S^{\mathbf{l}}$.

It follows from the previous results that $X$ has a periodic motion as a generic attractor if and only if there exists an admissible $D$ such that the trajectories of the complex extension of $\phi$ issuing from a suitable $C^{n}$-neighbourhood of $D$ remain uniformly bounded in the future. As a consequence, we also get the following corollary.

Corollary 1. A necessary and sufficient condition for $X$ to have a periodic motion as a generic attractor is the existence of a differentiable function $\lambda: N \rightarrow R\left(N\right.$ a $C^{n}$ 
open set), $\lambda(x)=0$ for $x \in N \cap R^{n}$, such that:

(1) The vector field $(1+\lambda i) X$ is defined on $N$ and there exists a compact $2 n$ dimensional differentiable submanifold $N$ with boundary $M \subset N$ such that at each point of the boundary of $M$, the vector field $(1+\lambda i) X$ points to the interior of $M$.

(2) $M \cap R^{n}$ contains an admissible set $D$.

(3) For some $k>0, \dot{\lambda} \lambda \leq-k \lambda^{2}$ for $x \in N$, where

$$
\dot{\lambda}(x)=(1+\lambda(x) i) X_{x}(\lambda)=\lim _{t \rightarrow 0} \frac{1}{t}\left(\lambda\left(x+t(1+\lambda(x) i) X_{x}\right)-\lambda(x)\right) .
$$

Proof. Sufficiency. Because of (1) the trajectories of $(1+\lambda i) X$ are bounded in the future. On the other hand it follows easily from (3) that if $x(t)$ is a trajectory of $(1+\lambda i) X, \lambda^{2}(x(t))=\mu(t)$ satisfies $\mu(t) \leq \mu(0) \exp (-2 k t)$ and hence $|\lambda(x(t))|$ $\leq|\lambda(x(0))| \exp (-k t)$. Consider the function $s(t)=\int_{0}^{t} \lambda(x(u)) d u$; clearly $|s(t)|$ $\leq|\lambda(x(0))| / k$ for $t \geq 0$.

The differential equation $\dot{s}=\lambda(\phi(x, t+i s))$ is defined for small $t$ and $s$ and if $s(t)$ is its solution with $s(0)=0$ it is easy to check that $\phi(x, t+i s(t))$ is the trajectory $x(t)$ of $(1+\lambda i) X$ with the initial condition $x(0)=x$. So, $s(t)$ $=\int_{0}^{t} \lambda(x(u)) d u$ and thus $|s(t)| \leq|\lambda(x)| / k$.

If $x \in M$ and is close enough to $R^{n}, \phi(x, t)$ cannot get far from $M$; otherwise, $x(t)$ would also be far from it, which is absurd on account of (1). Therefore the $\phi(x, t)$ are uniformly bounded on a $C^{n}$-neighbourhood of $D$ and in view of previous remarks this completes the proof of the sufficiency.

Necessity. Let $A$ be the periodic motion and $U$ a $C^{n}$-neighbourhood of $A$ such that for each $x \in U, \beta(x)$ ( $\beta$ denotes the complex extension of the retraction $\alpha$; see the proof of Theorem 4) can be written $\beta(x)=\phi(\bar{x}, t+i s)$ where $\bar{x} \in A$ and $s$ is a real number. Clearly $s$ is uniquely determined and we define $\lambda(x)$ as $-s(\beta(x))$. Then, the trajectories of $(1+\lambda i) X$ are $\phi\left(x, t+i \lambda(x)\left(1-e^{-t}\right)\right)$ as it is easy to prove owing to the commutativity between $\beta$ and the flow. Since $\lambda\left(\phi\left(x, t+i \lambda(x)\left(1-e^{-t}\right)\right)=\lambda(x) e^{-t}\right.$ it follows that $\dot{\lambda}(x)=-\lambda(x)$ or $\lambda \dot{\lambda} \leq-\lambda^{2}$ which implies (3). On the other hand, (1) is a consequence of the fact that $\phi\left(x, t+i \lambda(x)\left(1-e^{-t}\right)\right)$ converges to $A$ uniformly on a suitable $C^{n}$-neighbourhood of $A$. (See, for instance, [1].)

Other corollaries can also be obtained using the well-known results of Montel on normal families. For instance,

Corollary 2. Let $a_{i}, i=1,2, \ldots, n$ be positive real numbers. Then a necessary and sufficient condition for $X$ to have a periodic motion as a generic attractor is the existence of an admissible subset $D$ such that all the trajectories of the vector field $\left(\Pi_{1}^{n}\left(x_{i}^{2}+a_{i}\right)\right) X$ issuing from some $C^{n}$-neighbourhood of $D$ can be defined in the future.

Proof. Since on $R^{n}$ the flow defined by $\left(\prod_{1}^{n}\left(x_{i}^{2}+a_{i}\right)\right) X$ is just a reparametrization of $\phi$, the necessity follows at once from Theorem 1 . 
For the sufficiency, let us denote by $\bar{\phi}$ the flow defined by $\left(\Pi_{1}^{n}\left(x_{i}^{2}+a_{i}\right)\right) X$. If $U$ is a $C^{n}$-neighbourhood of $D$ such that the $\bar{\phi}_{t}$ are defined on it for all $t$ and such that no point with some $x_{i}= \pm i\left(a_{i}\right)^{1 / 2}$ belongs to it, then, for no $t, \bar{\phi}_{t}(U)$ intersects the hyperplanes $x_{i}= \pm i\left(a_{i}\right)^{1 / 2}$ that consist only of critical points of the vector field $\left(\Pi_{1}^{n}\left(x_{i}^{2}+a_{i}\right)\right) X$. Therefore by Montel's theorem the $\bar{\phi}_{t}$ constitute a normal family and being uniformly bounded on $D$ they are uniformly bounded on a suitable $C^{n}$-neighbourhood of it. Since, on $R^{n}, \phi$ is a reparametrization of $\bar{\phi}$, the result follows again from Theorem 1 .

6. Let $X$ be an analytic vector field, $A$ a $C$-generic attractor of the flow associated to $X, U$ an open and bounded positively invariant $R^{n}$-neighbourhood of $A$, and let $\alpha$ be the retraction of $U$ onto $A$ defined previously. In this and the following section we show, among other things, that if $Y$ is a vector field close to $X$ on $U$ (in a sense to be made precise later), then there exists a unique retraction $\gamma$, close to $\alpha$, of $U$ onto a compact submanifold of $U$ homeomorphic to $A$ that commutes with $Y$. These results are connected, in particular, with the perturbation theory of invariant manifolds. (See, for instance, [4].)

First, we study some geometrical properties of small functions defined on $R(\alpha)$ ( $R$ stands for range). The discussion that follows until and including Lemma 8 concerns those properties. Let $\delta_{0}>0$ be such that $B=\left\{x \in R^{n}:\|x-y\|\right.$ $\leq \delta_{0}$, for some $\left.y \in R(\alpha)\right\}$ is contained in $U$, and let $M>1$ be an upper bound for the norm of the matrix $\left(\left(\partial \alpha_{i} / \partial x_{j}\right)\left(x_{i, j}\right)\right)$ where $x_{i, j} \in B, i, j=1, \ldots, n$. Choose $\varepsilon>0$ such that $a=1-\varepsilon(2+M)>0$, and $\delta_{1}>0$ such that if $x \in R(\alpha)$ and $\left\|x_{i, j}-x\right\| \leq \delta_{1}, i, j=1, \ldots, n$, then $\left\|\alpha_{x}^{\prime}-\left(\left(\partial \alpha_{i} / \partial x_{j}\right)\left(x_{i, j}\right)\right)\right\|$ $\leq \varepsilon$. Also, take $\delta_{2}=\delta_{1} / 4$ and $\delta_{3}$ such that $M \delta_{3}<\delta_{2} / 3$.

Lemma 5. Let $f: R(\alpha) \rightarrow R^{n}$ be a continuous function such that $\|f\|$ $=\sup _{x \in R(\alpha)}\|f(x)\| \leq \delta_{3}$ and that for $x, y \in R(\alpha)$ and $\|x-y\| \leq \delta_{2}, \| f(x)$ $-f(y)\|\leq \varepsilon\| x-y \|$. Then the function $H_{f}: R(\alpha) \rightarrow R(\alpha), H_{f}(x)=\alpha(\dot{x}+f(x))$ is a homeomorphism onto $R(\alpha)$ and $\left\|H_{f}(x)-H_{f}(y)\right\| \geq a\|x-y\|$ for $x, y \in R(\alpha)$ and $\|x-y\| \leq \delta_{2}$.

Proof. We have that

$$
H_{f}(x)-H_{f}(y)=\alpha(x+f(x))-\alpha(y+f(y))=\alpha_{*}^{\prime}(x-y+f(x)-f(y)),
$$

where $\left(\alpha_{*}^{\prime}=\left(\partial \alpha_{i} / \partial x_{j}\right)\left(x_{i, j}\right)\right)$ for some $x_{i, j} \in U,\left\|x_{i, j}-x\right\| \leq \delta_{1}, i, j=1, \ldots, n$. On the other hand, since $\alpha(x)-\alpha(y)=x-y$, we have that $\alpha_{* *}^{\prime}(x-y)=x$ $-y$ where $\left\|\alpha_{* *}^{\prime}-\alpha_{x}^{\prime}\right\| \leq \varepsilon$. Thus $\alpha_{x}^{\prime}(x-y)=x-y+\left(\alpha_{x}^{\prime}-\alpha_{* *}^{\prime}\right)(x-y)$, and therefore

$$
\begin{aligned}
H_{f}(x)-H_{f}(y) & =\alpha_{x}^{\prime}(x-y)+\left(\alpha_{*}^{\prime}-\alpha_{x}^{\prime}\right)(x-y)+\alpha_{*}^{\prime}(f(x)-f(y)) \\
& =x-y+\left(\alpha_{x}^{\prime}-\alpha_{* *}^{\prime}\right)(x-y)+\left(\alpha_{*}^{\prime}-\alpha_{x}^{\prime}\right)(x-y)+\alpha_{*}^{\prime}(f(x)-f(y)),
\end{aligned}
$$


and

$$
\begin{aligned}
\left\|H_{f}(x)-H_{f}(y)\right\| & \geq\|x-y\|-\varepsilon\|x-y\|-\varepsilon\|x-y\|-M \varepsilon\|x-y\| \\
& =a\|x-y\| .
\end{aligned}
$$

To prove that $H_{f}$ is a homeomorphism onto the connected manifold $R(\alpha)$ it is enough to notice that, if $z \in R(\alpha),\left\|H_{f}(z)-z\right\|=\|\alpha(z+f(z))-z\| \leq M \delta_{3}$ $<\delta_{2}$. Hence if $x, y \in R(\alpha)$ and $\|x-y\| \geq \delta_{2}, H_{f}(x) \neq H_{f}(y)$.

Lemma 6. Let $f$ be as in the previous lemma and set $K_{f}=H_{f}^{-1}$. Then for every $x, y \in R(\alpha),\|x-y\| \leq \delta_{2}$, we have that $\left\|K_{f}(x)-K_{f}(y)\right\| \leq N\|x-y\|$, where $N=\max \left(1 / a, 2 \delta_{2} / \delta_{3}\right)$.

Proof. Let $\|x-y\| \leq \delta_{3}$; we have that $\|x-y\|=\left\|H_{f}\left(K_{f}(x)\right)-H_{f}\left(K_{f}(y)\right)\right\|$ $\geq a\left\|K_{f}(x)-K_{f}(y)\right\|$ since $\left\|K_{f}(x)-K_{f}(y)\right\| \leq\left\|K_{f}(x)-x\right\|+\|x-y\|+\| K_{f}(y)$ $-y \| \leq 2 M \delta_{3}+\delta_{3}<\delta_{2}$. Therefore $\left\|K_{f}(x)-K_{f}(y)\right\| \leq(1 / a)\|x-y\|$.

Assume now that $\|x-y\| \leq \delta_{2}$ and $\|x-y\| \geq \delta_{3}$. Then

$$
\frac{\left\|K_{f}(x)-K_{f}(y)\right\|}{\|x-y\|} \leq \frac{\delta_{2}+\frac{2}{3} \delta_{2}}{\delta_{3}}<2 \frac{\delta_{2}}{\delta_{3}}
$$

which completes the proof.

Let $P=\max ((N+3), N+\varepsilon N+1)$ and define the function $p$ by $p(f)=\psi$, where $\psi(x)=K_{f}(x)+f\left(K_{f}(x)\right)-x$ for $x \in R(\alpha)$.

Lemma 7. Let $f$ be as in Lemma 5. Then $\|\psi\|<\delta_{2}$ and $\|\psi(x)-\psi(y)\|$ $\leq P\|x-y\|$ for $x, y \in R(\alpha)$ and $\|x-y\| \leq \delta_{2}$.

Proof. $\|\psi(x)\| \leq\left\|K_{f}(x)-x\right\|+\left\|f\left(K_{f}(x)\right)\right\| \leq M \delta_{3}+\delta_{3}<\delta_{2}$. If $\|x-y\|$ $\leq \delta_{3}$,

$$
\left\|K_{f}(x)-K_{f}(y)+f\left(K_{f}(x)\right)-f\left(K_{f}(y)\right)+y-x\right\| \leq(N+\varepsilon N+1)\|x-y\| .
$$

If $\|x-y\| \leq \delta_{2}$, and $\|x-y\| \geq \delta_{3}$,

$$
\|\psi(x)-\psi(y)\| /\|x-y\| \leq N+2 \delta_{3} / \delta_{3}+1=N+3 .
$$

Hence, for $\|x-y\| \leq \delta_{2}$, we have that $\|\psi(x)-\psi(y)\| \leq P\|x-y\|$.

Lemma 8. Let $f, g$ satisfy the assumptions of Lemma 5. Then

$$
\|p(f)-p(g)\| \leq(M / a+\varepsilon M / a+1)\|x-y\| .
$$

Proof. Put $\psi=p(f), \phi=p(g)$. We have that

$$
\begin{aligned}
\psi(x)-\phi(x) & =K_{f}(x)+f\left(K_{f}(x)\right)-K_{g}(x)-g\left(K_{g}(x)\right) \\
& =K_{f}(x)-K_{g}(x)+f\left(K_{f}(x)\right)-f\left(K_{g}(x)\right)+(f-g)\left(K_{g}(x)\right),
\end{aligned}
$$


which implies

$$
\|\psi(x)-\phi(x)\| \leq\left\|K_{f}(x)-K_{g}(x)\right\|+\varepsilon\left\|K_{f}(x)-K_{g}(x)\right\|+\|f-g\| .
$$

On the other hand, from $H_{f}\left(K_{f}(x)\right)-H_{g}\left(K_{f}(x)\right)+H_{g}\left(K_{f}(x)\right)-H_{g}\left(K_{g}(x)\right)=0$, we get that

$$
\begin{aligned}
a \| K_{f}(x)- & K_{g}(x)\|\leq\| H_{g}\left(K_{f}(x)\right)-H_{g}\left(K_{g}(x)\right) \| \\
& =\left\|H_{f}\left(K_{f}(x)\right)-H_{g}\left(K_{g}(x)\right)\right\| \leq M\left\|f\left(K_{f}(x)\right)-g\left(K_{f}(x)\right)\right\| \leq M\|f-g\| .
\end{aligned}
$$

Consequently, $\left\|K_{f}(x)-K_{g}(x)\right\| \leq(M / a)\|f-g\|$, and we obtain finally that

$$
\|\psi(x)-\phi(x)\| \leq(M / a+\varepsilon M / a+1)\|f-g\| .
$$

Let $\mathcal{\exists}$ be the space of the continuous functions $\psi: R(\alpha) \rightarrow R^{n}$ such that $\alpha(x+\psi(x))=x, \quad x \in R(\alpha) ; \sup _{x \in R(\alpha)}\|\psi(x)\| \leq \delta_{0}$, and $\|\psi(x)-\psi(y)\| \leq$ $P\|x-y\|$ for $x, y \in R(\alpha),\|x-y\| \leq \delta_{2}$. If we define, for $\psi, \phi \in \mathcal{\exists}$, $d(\psi, \phi)=\sup _{x \in R(\alpha)}\|\psi(x)-\phi(x)\|,(\mathcal{G}, d)$ becomes a compact metric space. Let w: $U \rightarrow U$ be a continuous function such that

$$
\sup _{x \in U}\|(w-\alpha)(x)\| \leq \delta_{3}
$$

and

$$
\|(w-\alpha)(x)-(w-\alpha)(y)\| \leq Q\|x-y\| \text { for } x, y \in U
$$

where $Q>0$, and $Q(1+P)<\varepsilon, Q(M / a+\varepsilon M / a+1)<1$.

For $\psi \in \mathcal{I}$ define $\Omega(\psi)=f$, where $f(x)=w(x+\psi(x))-x, x \in R(\alpha)$. Now we can state

Theorem 5. $p \circ \Omega$ is a contraction of 9 .

Proof. First we show that $P \circ \Omega$ is a mapping from $\Im$ to $\Im$. For $x \in R(\alpha)$ we have that $w(x+\psi(x))-x=w(x+\psi(x))-\alpha(x+\psi(x))$. Thus, $\|f\|=\|(w-\alpha)$ $\cdot(x+\psi(x)) \| \leq \delta_{3}$.

On the other hand, if $\|x-y\| \leq \delta_{2}$,

$$
\begin{aligned}
\|f(x)-f(y)\| & =\|(w-\alpha)(x+\psi(x))-(w-\alpha)(y+\psi(y))\| \\
& \leq Q(\|x-y\|+\|\psi(x)-\psi(y)\|) \leq \varepsilon\|x-y\| .
\end{aligned}
$$

Hence $f$ satisfies the assumptions of Lemma 5, and since $\alpha(x+p(f)(x))$ $=\alpha\left(K_{f}(x)+f\left(K_{f}(x)\right)\right)=H_{f}\left(K_{f}(x)\right)=x$, it follows that $p(f) \in \Im$.

Next we prove that $p \circ \Omega$ is a contraction. Let $\psi, \phi$ belong to $\exists$; then we may apply Lemma 8 to $f=\Omega(\psi)$ and $g=\Omega(\phi)$ to get 


$$
d(p \circ \Omega(\psi), p \circ \Omega(\phi)) \leq(M / a+\varepsilon M / a+1)\|f-g\| .
$$

But

$$
\begin{aligned}
\|f-g\| & =\|w \circ(\alpha+\psi)-w \circ(\alpha+\phi)\| \\
& =\|(w-\alpha) \circ(\alpha+\psi)-(w-\alpha) \circ(\alpha+\phi) \mid \leq Q d(\psi, \phi) .
\end{aligned}
$$

Consequently if $w$ satisfies the conditions (*),p,$\Omega$ is a contraction of $\exists$.

Corollary 3. If $w$ satisfies the conditions (*), there exists a manifold $V$ homeomorphic to $R(\alpha)$ that is invariant under $w$.

Proof. Let $\psi$ be the fixed point of $p \circ \Omega$, and take $V=\{z \in U: z=x$ $+\psi(x)$ for some $x \in R(\alpha)$ \}. The function $x \rightarrow x+\psi(x)$ is a homeomorphism of $R(\alpha)$ onto $V$ since $x+\psi(x)=y+\psi(y)$ implies $x=\alpha(x+\psi(x))=\alpha(y+\psi(y))$ $=y$. Now, let us show that $w(x+\psi(x)) \in V$ for every $x \in R(\alpha)$. Let $w(x+\psi(x))$ - $x$ be denoted again by $f$; since $\psi=p(f)$ we know that $\psi\left(H_{f}(x)\right)=K_{f}\left(H_{f}(x)\right)$ $+f\left(K_{f}\left(H_{f}(x)\right)\right)-H_{f}(x)$, i.e. $H_{f}(x)+\psi\left(H_{f}(x)\right)=x+f(x)=w(x+\psi(x))$. Since $H_{f}(x)+\psi\left(H_{f}(x)\right) \in V$, this completes the proof.

On account of the compactness of $\exists$ it is easy to conclude that for any $\delta>0$ there is a positive integer $n$ such that the $n$-iterate of $p \circ \Omega$ applied to any $\phi \in \mathcal{Y}$ is $\delta$-close to the fixed point $\psi$ of $p \circ \Omega$. Since, on the other hand, for each $y$ near $V$ there exists $\phi \in \exists$ such that $y=x+\phi(x)$ for some $x \in R(\alpha)$, we obtain that $V$ is an attractor (as defined in the introduction) of the discrete flow $\Phi(x, n)$ $=w^{n}(x), x \in U, n=1,2, \ldots$. Consequently if $D$ is an invariant set of $w$ lying near $V$ and $w(D)=D$, then $D \subset V$. In particular, if $w$ is a homeomorphism and $D$ is a compact connected manifold of the dimension of $V$, then $D=V$. Hence, if $\pi: U \rightarrow U$ is a homeomorphism close to the identity that commutes with $w$, we obtain that $V$ is also invariant under $\pi$ since $\pi(V)$ is a compact connected manifold and $w(\pi(V))=\pi(w(V))=\pi(V)$. These remarks together with previous results imply readily the following corollary.

Corollary 4. There is a $C^{1}(U)$ neighbourhood of $X$ such that if $Y$ belongs to it, the flow defined by $Y$ has an attractor $V \subset U$ homeomorphic to $A$.

Perhaps it is worthwhile to say that as a consequence of the implicit function theorem it may be shown that there is a compact neighbourhood of $\psi=0$ in $\exists$ with a convex structure. The existence of invariant manifolds may also be obtained as an application of Schauder's fixed point theorem.

If instead of $\Im$ we consider a space $\Im_{1}$ of continuous functions $\psi: R(\alpha) \rightarrow R^{n}$, $\alpha(x+\psi(x))=x$, that have continuous partial derivatives satisfying a uniform Lipschitz condition, we get with similar arguments the existence of an invariant $C^{\prime}$ manifold for any function $w: U \rightarrow U, C^{1}(U)$ close to $\alpha$ and such that $(w-\alpha)^{\prime}$ has a small Lipschitz constant on $U$. 
7. Consider again the space 3 . It is clear from its definition that there exists $\bar{P} \geq P$ such that $\|\psi(x)-\psi(y)\| \leq \bar{P}\|x-y\|$ for each $x, y \in R(\alpha)$ and each $\psi \in \mathcal{T}$. Let $L>0$ be such that

$$
\|(\alpha+\psi \circ \alpha)(x)-(\alpha+\psi \circ \alpha)(y)\| \leq L\|x-y\|
$$

for each $x, y \in U$ and each $\psi \in \mathcal{Y}$. We want to show that if $w: U \rightarrow U$ is a homeomorphism satisfying (*) with a $Q$ also less than $[2(\bar{P}+1)(L+1)]^{-1}$, then there is a retraction of $U$ onto $V$ (the invariant manifold of $w$ ) that commutes with $w$.

Let $\psi: R(\alpha) \rightarrow R^{n}, \alpha(x+\psi(x))=x$, be such that $V=\{x+\psi(x): x \in R(\alpha)\}$, and define $\beta: U \rightarrow U$ by $\beta(x)=\alpha(x)+(\psi \circ \alpha)(x)$. Then $\beta$ is a retraction, for $\beta \circ \beta=\alpha \circ \beta+\psi \circ \alpha \circ \beta=\beta$ since $\alpha \circ \beta=\alpha$. We also have from the invariance of $R(\beta)=V$ under $w$ that $\beta \circ w \circ \beta=w \circ \beta$.

Let $\mathcal{L}$ be the space of continuous functions $\lambda: U \rightarrow R^{n}$ such that $\sup _{x \in U, x \neq \beta(x)}\|\lambda(x)\| /\|x-\beta(x)\|<\infty, \lambda \circ \beta=0$, and $\beta \circ(\beta+\lambda)=\beta+\lambda$. For $\lambda, \mu \in \mathcal{L}$, set

$$
d(\lambda, \mu)=\sup _{x \in U, x \neq \beta(x)}\|(\lambda-\mu)(x)\| /\|x-\beta(x)\| .
$$

Then, with $d$ as the distance, $\mathcal{L}$ becomes a complete metric space. Let $w$ satisfy (*) with such a $Q$ that $2 Q(\bar{P}+1)(L+1)<1$ and for $\lambda \in \mathcal{L}$, set $O(\lambda)=w^{-1}$ $\circ(\beta+\lambda) \circ w-\beta$. Since $(\beta+\lambda) \circ w \circ \beta=w \circ \beta$ we have that $O(\lambda)=0$; we also have that $\beta \circ(\beta+O(\lambda))=\beta+O(\lambda)$ as may be verified immediately.

Theorem 6. $O$ is a contraction of $\mathcal{L}$.

Proof. If $x, y \in R(\beta)$ we have $x-y=\alpha(x)-\alpha(y)+\psi(\alpha(x))-\psi(\alpha(y))$, which implies $\|x-y\| \leq\|\alpha(x)-\alpha(y)\|+P\|\alpha(x)-\alpha(y)\|$ and $\|\alpha(x)-\alpha(y)\|$ $\geq(1+\bar{P})^{-1}\|x-y\|$. Then

$$
\begin{aligned}
\|w(x)-w(y)\| & \geq\|\alpha(x)-\alpha(y)\|-\|(w-\alpha)(x)-(w-\alpha)(y)\| \\
& \geq\left(\frac{1}{\bar{P}+1}-\frac{1}{2(\bar{P}+1)}\right)\|x-y\|=\frac{1}{2(\bar{P}+1)}\|x-y\| .
\end{aligned}
$$

Therefore, since $x-y=w\left(w^{-1}(x)\right)-w\left(w^{-1}(y)\right)$, we obtain that for $x, y$ $\in R(\beta),\left\|w^{-1}(x)-w^{-1}(y)\right\| \leq 2(\bar{P}+1)\|x-y\|$.

Let $\lambda, \mu \in \mathcal{L}$. From $O(\lambda)-O(\mu)=w^{-1} \circ(\beta+\lambda) \circ w-w^{-1} \circ(\beta+\mu) \circ w$, we get that

$$
\|O(\lambda)(x)-O(\mu)(x)\| \leq 2(\bar{P}+1)\|\lambda(w(x))-\mu(w(x))\|,
$$

or

$$
\|O(\lambda)(x)-O(\mu)(x)\| \leq 2(\bar{P}+1)\|(\lambda-\mu)(w(x))\| .
$$


Consequently

$$
\begin{aligned}
\| O(\lambda)(x)- & O(\mu)(x)\|\leq 2(\bar{P}+1) d(\lambda, \mu)\| w(x)-\beta(w(x)) \| \\
& \leq 2(\bar{P}+1) d(\lambda, \mu)(\|w(x)-w(\beta(x))\|+\|\beta(w(\beta(x)))-\beta(w(x))\|) \\
& \leq 2(\bar{P}+1)(L+1) d(\lambda, \mu)\|w(x)-w(\beta(x))\| \\
& =2(\bar{P}+1)(L+1) d(\lambda, \mu)\|(w-\alpha) x-(w-\alpha)(\beta(x))\| .
\end{aligned}
$$

Thus, $d(O(\lambda), O(\mu)) \leq 2(\bar{P}+1)(L+1) Q d(\lambda, \mu)$, which implies the thesis of the theorem.

If $\lambda$ is the fixed point of $O$, we have that $(\beta+\lambda) w=w(\beta+\lambda)$ and as $(\beta+\lambda) \circ(\beta+\lambda)=\beta \circ(\beta+\lambda)+\lambda \circ(\beta+\lambda)=(\beta+\lambda)+\lambda \circ \beta \circ(\beta+\lambda)$ $=\beta+\lambda, \beta+\lambda$ is a retraction of $U$ onto $V$.

Corollary 5. If $Y$ is a vector field close enough to $X$ in the $C^{\prime}(U)$ topology, there is a retraction $\gamma$ of $U$ onto a manifold $V$ that commutes with $Y$.

Proof. Call $\Psi$ the flow determined by $Y$, and let $T>0$ be such that $w=\Psi_{T}$ satisfies the assumptions of the previous theorem. If $\gamma$ is the retraction that commutes with $w$, then $\Psi_{-t} \gamma \Psi_{t}-\beta \in \mathcal{L}$ for small $|t|$, since, as we have already shown, $R(\gamma)$ is invariant under $\Psi_{t}$. But $w^{-1} \circ \Psi_{-t} \circ \gamma \circ \Psi_{t} \circ w=\Psi_{-t} \circ \gamma \circ \Psi_{t}$ and on account of the uniqueness of the fixed point of $O$, we must have $\Psi_{-t} \circ \gamma \circ \Psi_{t}$ $=\gamma$ as had to be shown.

The uniqueness of $\gamma$ as a retraction close to $\alpha$ that commutes with $Y$ follows at once from the uniqueness of the manifold $R(\gamma)$.

As before, the differentiable case may be handled in a very similar way. However, the space $\mathcal{L}_{1}$ to be considered in that case should consist of the $C^{\mathbf{l}}$. functions $\lambda, \lambda \circ \beta=0, \beta \circ(\beta+\lambda)=\beta+\lambda$, such that

$$
\sup _{x \in U, x \neq \beta(x)} \frac{\left|\lambda^{\prime}(x)\right|}{\|x-\beta(x)\|}<\infty
$$

where $\left|\lambda^{\prime}(x)\right|=\sup _{,=\beta^{\prime}(x),\|,\| \|=1}\left\|\lambda^{\prime}(x) v\right\|$ and the metric $d$ for $\mathcal{L}_{1}$ should be $d=d_{1}+d_{2}$, where $d_{1}(\lambda, \mu)=\sup _{x \in U}\|(\lambda-\mu)(x)\|+\sup _{x \in U}\left\|(\lambda-\mu)^{\prime}(x)\right\|$, and

$$
d_{2}(\lambda, \mu)=\sup _{x \in U, x \neq \beta(x)} \frac{\left|(\lambda-\mu)^{\prime}(x)\right|}{\|x-\beta(x)\|} .
$$

Remark. A generic periodic saddle point of an analytic vector field $X$ need not have $C^{2}$-retractions that commute with $X$. If such a retraction exists it is possible to define in a neighbourhood of the periodic motion a closed $C^{2} 1$-form $w$, such that $w(X)=1$. Indeed, if $r$ is the retraction onto the closed orbit $p$, consider on $p$ the form $d t, d t(X)=1$; then $w=r^{*} d t$ is obviously a closed form and satisfies $w(X)=d t\left(r^{\prime}(X)\right)=1$. Consider in $R^{2} \times S^{1}$ the flow given be $\varphi\left(x, y, e^{i \theta}, t\right)$ 
$=\left(x e^{-t}, y e^{t / n}, e^{i \theta} e^{2 \pi i t}\right)$, where $n$ is a positive integer. The retraction $r, r\left(x, y, e^{i \theta}\right)$ $=\left(0,0, e^{i \theta}\right)$, commutes with the flow, and hence there exists a closed 1-form $w$, analytic in a neighbourhood of $p=\{(0,0)\} \times S^{1}$ such that $w(X)=1, X$ being the vector field defined by the flow. Let $f\left(x, y, e^{i \theta}\right)=1+a x y^{n}, a>0$, and consider the flow associated to the vector field $f X$ that has a generic periodic saddle point at $(0,0,1)$. If there were a closed 1 -form $w^{\#}$ such that $w^{\#}(f X)=1$, then, since $\int_{p} w^{\#}=\int_{p} w=1$, we would have $w^{\#}=w+d h, h$ being a $C^{2}$ real function defined in a neighbourhood of $p$. From $1=w^{\#}(f X)=f(1+X(h))$ we get that $X(h)=-a x y^{n}\left(1+a x y^{n}\right)^{-1}$. Therefore

$$
h\left(x / e_{n+1}, y e^{1 / n}, 1\right)-h(x, y, 1)=-a x y^{n}\left(1+a x y^{n}\right)^{-1} ;
$$

however, if we take $\partial^{n+1} / \partial x \partial y^{n}$ on both sides and evaluate at $(0,0)$, we get $0=-a$. Thus, if we take $n=1, f X$ has no $C^{2}$ retraction commuting with it.

This fact may be used to show that there are $C$-generic attractors $A$ of analytic vector fields $X$ such that we can find a complex neighbourhood $U$ of $A$ and a sequence $\left\{X_{n}\right\}$ of analytic vector fields that converges uniformly to $X$ on $U$, and so that there is no analytic retraction onto the invariant manifold $A_{n}$ of $X_{n}$ that commutes with $X_{n}$. (The periodic motion of the above example may lay on $A_{n}$.)

\section{REFERENCES}

1. J. L. Massera, On Liapounoffs conditions of stability, Ann. of Math. (2) 50 (1949), 705-721. MR $11,721$.

2. J. W. Milnor, Topology from the differentiable viewpoint, University Press of Virginia, Charlottesville, Va., 1965. MR 37 \#2239.

3. D. Montgomery and L. Zippin, Topological transformation groups, Interscience, New York, 1955. MR 17, 383.

4. R. Sacker, A new approach to the perturbation theory of invariant surfaces, Comm. Pure Appl. Math. 18 (1965), 717-732. MR 32 \#6003.

5. S. Smale, Stable manifolds for differential equations and diffeomeophisms, Ann. Scuola Norm. Sup. Pisa (3) 17 (1963), 97-116. MR 29 \#2818b.

Instituto de Matematica PURa E Aplicada Rio de Janeiro, Brasil

Instituto de Mathematica y Estadistica, Montevideo, Uruguay

Current address: Instituto de Matemática y Estadística, Avda. J. Herrera y Reissig 565, Montevideo, Uruguay 